\title{
Incubation and its implications for the interpretation of the ECS gradient effect '
}

\author{
J. P. J. PINEL2 AND R. M. COOPER \\ UNIVERSITY OF CALGARY
}

Rats were given one-trial avoidance training followed by an electroconvulsive shock (ECS) at various intervals and a test of retention $25 \mathrm{hr}$. later. The ECS gradient effect was obtained, that is, the sooner after learning the ECS was administered, the more retention was disrupted. In a second experiment, it was shown that a one-trial avoidance response increased in strength over time. Implications of this incubation effect for the interpretation of the ECS gradient effect are discussed.

The one-trial avoidance task has been used extensively in the study of the amnesic effects of electroconvulsive shock (ECS). The ECS gradient effect, that is, the demonstration that the sooner an ECS is administered after learning the more retention will be dismupted, has been demonstrated after one-trial avoidance training by a number of investigators (Heriot \& Coleman, 1962; King, 1965; and others). However, these investigators have typically not included control groups in their experiments which would reveal the normal retention function of the one-trial avoidance response. Pinel \& Cooper (1966) have pointed out that until more is known about the retention function after one-trial avoidance learning the interpretation of the effects of ECS on this function will continue to be difficult.

Several investigators (Brady, 1951; McMichael, 1965; and others) have shown that some avoidance responses increase in strength or incubate over time. If one-trial avoidance responses also incubate, an alternative to the usual consolidation interpretation of the ECS gradient effect can be offered. According to the consolidation theory there is an increase in the permanence of memory storage over time; however, no corresponding increase in response strength per se is implied.

ECS has been reported to have a greater disruptive effect on weaker responses (Braun \& Albee, 1952). Thus, when an ECS is administered shortly after training, the disruptive effect may be great, not because the engram has yet to be transferred from temporary to permanent storage, but because the learned response has not had a chance to incubate.

\section{Procedure}

EXPERIMENT 1

Thirty-two experimentally naive, male, black-hooded rats were trained to drink from a spout located just above a large bar which was invariably depressed when Ss drank. Water was not contingent on a bar press, but the bar enabled a record of drinking behavior to be kept. On the last preshock training trial, the median latency, that is, the amount of time. it took Ss to depress the bar after being placed in the test box was $3 \mathrm{sec}$. On the avoidance training day, Ss received a $12 \mathrm{ma}$ DC shock (calibration resistance $=100 \mathrm{~K}$ ) the first time they pressed the bar and were immediately removed from the box. Three groups of nine Ss each received an ECS 30 sec., 2 min., or $4 \mathrm{hr}$. after avoidance training. These Ss, as well as an additional five control Ss who received no ECS, were given retention tests $25 \mathrm{hr}$. afterward. ECS consisted of a 0.3-sec. $50 \mathrm{ma} \mathrm{AC}$ current passed via saline-soaked, gauze-covered alligator clips attached to Ss' ears. The Ss were habituated to ear clips by applying them briefly after preshock training trials. All Se were deprived of water for $23 \mathrm{hr}$. prior to the last preshock training trial and to the postshock test of retention.

Results

Figure 1 indicates that the typical ECS gradienteffect was obtained. Animals that received an ECS $30 \mathrm{sec}$. after avoidance training had significantly shorter latencies on the test of retention than animals that received an ECS $2 \mathrm{~min}$. $(U=19, \mathrm{p}<.05)$ or $4 \mathrm{hr} .(\mathrm{U}=18, \mathrm{p}<.05)$ afterward. In addition, the $30-\mathrm{sec}$. Ss had significantly shorter latencies than the no-ECS control group $(U=8$, $\mathrm{p}<.05)$.

\section{EXPERIMENT 2}

\section{Procedure}

Animals received similar one-trial avoidance training to that used in Experiment 1. The median latency of

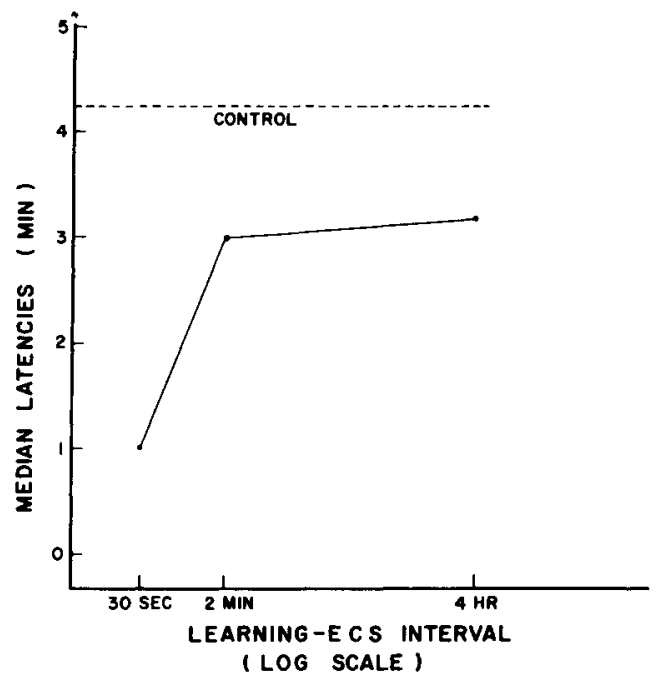

Fig. 1. ECS gradient effect. 


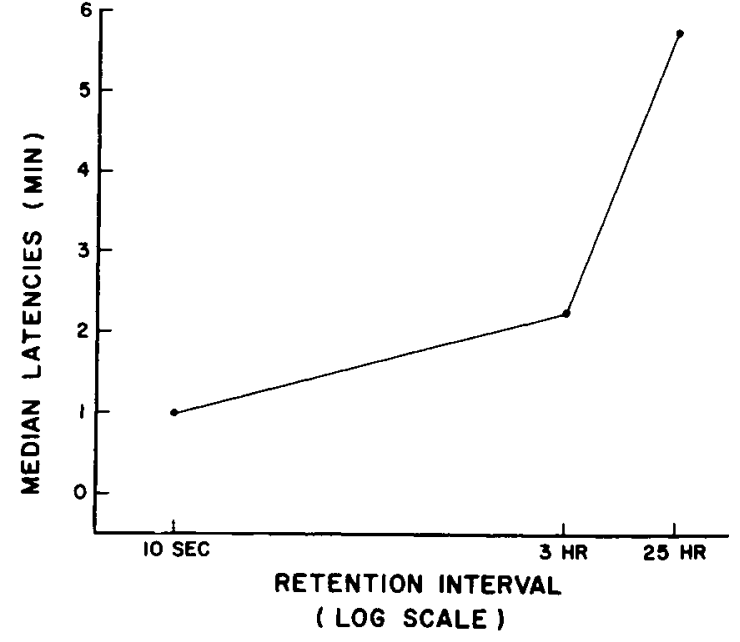

Fig. 2. Incubation effect.

Ss on the last preshock training trial was $3 \mathrm{sec}$. Of 16 Ss that were used, five were returned to the box for a test of retention $10 \mathrm{sec}$. after avoidance training, whereas five were returned at $3 \mathrm{hr}$. and six were returned at $25 \mathrm{hr}$.

\section{Results}

It can be seen from Fig. 2 that the one-trial avoidance response appeared to increase in strength or incubate over time. The latencies of the $25-\mathrm{hr}$. group were significantly greater than the latencies of both the 10-sec. $(U=1, p<.05)$ and $3-h r .(U=4, p<.05)$ groups.

\section{DISCUSSION}

The fact that the incubation and gradienteffects occur after similar one-trial avoidance training supports our hypothesis that the ECS gradient effect may be attributable to the incubation of the avoidance response. Perhaps, ECS administered $2 \mathrm{~min}$. or $4 \mathrm{hr}$. after learning had little effect on the strength of the avoidance response because incubation had occurred. On the other hand, the disruptive effects of an ECS administered 10 sec. after learning may be attributable to the fact that at this point little incubation had occurred, and the response was consequently weak and easily disrupted.

Pinel \& Cooper (1966) have also demonstrated a U-shaped retention function after one-trial avoidance training. However, in this earlier work lower shock levels and weaker habits were employed. In this regard,
Irwin \& Benuazizi (1966) have also reported a biphasic time-response curve for one-trial avoidance learning. In any case, in a series of studies similar to Experiment 2, we have consistently found an incubation effect under conditions in which we were able to demonstrate an ECS gradient effect.

Brady (1952) demonstrated that ECSs administered after emotional conditioning resulted in a gradient effect over a period of months. Adding support to our notion is the fact that Brady (1951) found that the conditioned emotional response that he employed incubated over a similar period of time.

The findings of Experiments 1 and 2 that both incubation and gradient effects occur after one-trial avoidance training gives support to our interpretation of the ECS gradient effect. However, there are considerable differences in the shape of the gradient and incubation curves suggesting that the relationship may be a spurious one. These differences, however, may be attributable to differences between the two experiments in experimental procedure. Before much consideration should be given to our hypothesis, gradient and incubation effects should be demonstrated in a common experiment so that more precise comparisons can be made.

\section{References}

Brady. J. V. The effect of electro-convulsive shock on a conditioned emotional response: the permanence of the effect. $J$. comp. physiol. Psychol., 1951, 44, 507-511.

Brady, J. V. The effect of electro-convulsive shock on a conditioned emotional response: the significance of the interval between the emotional conditioning and the electro-convulsive shock. J. comp. physiol. Psychol., 1952, 45, 9-13.

Braun, H. W., \& Albee, G. W. The relation between retention after electro-shock convulsions and degree of learning in the rat. J. comp. physiol. Psychol, , 1952, 45, 14-17.

Heriot, J. T., \& Coleman, P. D. The effect of electroconvulsive shock on retention of a modified "one-trial" conditi oned avoidance. J. comp. physiol. Psychol., 1962, 55, 1082-1084.

Irwin, S., \& Benuazizi, A. Pentylenetetrazol enhances memory function. Science, 1966, 152, 100-102.

King, R. A. Consolidation of the neural trace in memory: investigation with one-trial avoidance conditioning and ECS. J. comp. physiol. Psychol., 1965, 59, 283-295.

McMichael, J. S. Incubation of anxiety and instrumental behavior. J. comp. physiol. Psychol., 1966, 61, 208.211.

Pinel, J. P. J., \& Cooper, R. M. Demonstration of the Kamin effect after one-trial avoidance learning. Psychon. Sci., 1966, 4, 17-18.

\section{Notes}

1. Supported by Canadian National Research Council grant No. AP A-135.

2. Holder of a Canadian National Research Council Studentship. 